\title{
Estabilidade prática de sistemas afins com comutação a tempo discreto e ponto de equilíbrio parcialmente conhecido ${ }^{\star}$
}

\author{
Lucas N. Egidio* Grace S. Deaecto* \\ * Faculdade de Engenharia Mecânica, Unicamp \\ 13083-860, Campinas, São Paulo, Brasil \\ e-mails: \{egidio, grace\}@fem.unicamp.br
}

\begin{abstract}
In this paper, we present practical stability conditions for discrete-time switched affine systems based on Lyapunov-Metzler inequalities, which encompass others from the literature. These systems are used to model the dynamic behavior of several electronic devices, mainly in the power electronics domain. More specifically, the proposed conditions are less conservative for the existence of a set of attraction when compared with those derived from a quadratic Lyapunov function. Moreover, the proposed methodology allows for a convergence region dependent on the controlled output defined by the designer and, differently from many available techniques, admits that the equilibrium point be partially known. Two examples are used for illustration, being the first one composed of a switched affine system with no stable convex combination among its subsystems and the second, a practical application where the output voltage of a DC-DC boost converter is controlled.

Keywords: Switched affine systems, Lyapunov-Metzler inequalities, partial information of the equilibrium point

Resumo: Neste artigo, apresentamos condições de estabilidade prática para sistemas afins com comutação a tempo discreto baseadas em desigualdades de Lyapunov-Metzler, que são mais abrangentes do que outras presentes na literatura. Estes sistemas são utilizados para modelar o comportamento dinâmico de diversos dispositivos eletrônicos, principalmente na área de eletrônica de potência. Mais especificamente, as condições propostas são menos conservadoras para a existência de um conjunto de atração quando comparadas àquelas derivadas de uma função de Lyapunov quadrática. Nossas condições levam em conta uma região de convergência com base na saída controlada definida pelo projetista e, diferente de muitas técnicas disponíveis, permitem que o ponto de equilíbrio seja parcialmente conhecido. Dois exemplos são usados para ilustração da técnica de controle, sendo o primeiro composto por um sistema afim com comutação que não admite combinação convexa estável de seus subsistemas e o segundo, um exemplo de aplicação prática no qual a tensão de saída de um conversor CC-CC do tipo boost é controlada. Palavras-chaves: Sistemas afins com comutação, desigualdades de Lyapunov-Metzler, informação parcial do ponto de equilíbrio
\end{abstract}

\section{INTRODUÇÃO}

Sistemas com comutação constituem uma classe especial de sistemas híbridos, caracterizados por possuírem um número finito de subsistemas e uma regra de comutação que comanda o chaveamento entre eles. Vários sistemas de engenharia com dinâmicas complexas podem ser modelados como sistemas com comutação. De fato, estes sistemas são encontrados em diversas aplicações como, por exemplo, eletrônica de potência (veja Cardim et al. (2009) e Deaecto et al. (2010)), indústria automotiva (veja Camara et al. (2008)), controle em rede (veja Hespanha et al. (2007)),

\footnotetext{
* Este artigo é resultado de pesquisa científica apoiada pela "Coordenação de Aperfeiçoamento de Pessoal de Nível Superior (CAPES)", Código 001, pelo "Conselho Nacional de Desenvolvimento Científico e Tecnológico (CNPq)", processo 303499/2018-4 e pela "Fundação de Amparo à Pesquisa do Estado de São Paulo (FAPESP)", processo 2017/20343-0.
}

entre outras. Ademais, do ponto de vista teórico, apresentam características intrínsecas que permitem a obtenção de trajetórias estáveis mesmo no caso em que todos os subsistemas são instáveis, além de aprimorar a performance sempre que a regra de comutação for estritamente consistente, veja Deaecto et al. (2013). As referências Liberzon (2003), Shorten et al. (2007) e Sun and Ge (2005) são de grande relevância neste tema.

Nesse contexto, uma importante subclasse é aquela dos sistemas afins com comutação que, dentre outros, podem modelar diversos dispositivos de eletrônica de potência, veja Egidio et al. (2017) como exemplo de implementação prática nesta área. Estes sistemas apresentam um termo afim em suas equações dinâmicas que é responsável pela existência de um conjunto de pontos de equilíbrio atingíveis, compondo uma região no espaço de estado. Quando este termo afim é nulo o sistema se torna linear, caso para 
o qual a literatura apresenta condições de estabilidade em ambos domínios de tempo incluindo projeto de controle por realimentação de estado e de saída (e.g. Geromel and Colaneri (2006), Deaecto et al. (2011) e Fiacchini et al. (2016)). Já o caso afim é mais complexo e consiste em projetar uma regra de comutação para guiar as trajetórias do estado em direção a um ponto de equilíbrio de interesse que, de forma geral, não coincide com os pontos de equilíbrio de nenhum dos subsistemas isolados. Neste caso, a estabilidade assintótica geralmente só é garantida através do surgimento de modos deslizantes estáveis, o que implica em uma alta frequência de comutação. Para sistemas a tempo contínuo, a literatura apresenta resultados recentes para o controle por realimentação de estado e de saída, utilizando funções de Lyapunov quadráticas, veja Bolzern and Spinelli (2004), Deaecto and Santos (2015), Deaecto (2016) ou do tipo máximo, veja Scharlau et al. (2014). Contudo, a frequência de comutação necessária é arbitrária, fator que pode ser impeditivo em várias aplicações práticas, visto que ignora características naturais como tempo de resposta de chaves reais ou período de amostragem. Por este motivo, obter funções de comutação dotadas de um limitante superior para a frequência de chaveamento é um problema que tem ganhado interesse da comunidade científica. Nestes casos, as condições existentes na literatura asseguram estabilidade prática, onde as trajetórias são atraídas para uma região tão pequena quanto possível que contém o ponto de equilíbrio. Neste contexto, sistemas a tempo discreto ou com dados amostrados são classes de grande interesse, pois possuem a frequência de comutação naturalmente limitada. As referências Deaecto and Geromel (2017), Hetel and Fridman (2013), Xu and Zhai (2005) fornecem condições de estabilidade baseadas em uma função de Lyapunov quadrática, enquanto a referência Egidio and Deaecto (2019) é a pioneira em utilizar uma função de Lyapunov do tipo mínimo neste contexto.

Este artigo apresenta generalizações dos resultados obtidos em Egidio and Deaecto (2019) de forma a garantir estabilidade prática de um ponto de equilíbrio de interesse que seja apenas parcialmente conhecido, livrando o projetista da necessidade de conhecer a priori todas as suas componentes. Além disso, a otimização do conjunto de atração não é feita de forma a minimizar o volume de um elipsoide de atração, mas sim de uma bola no espaço vetorial definido pela saída controlada, permitindo que o projeto leve em conta uma otimização seletiva das variáveis de interesse. Assim como em Egidio and Deaecto (2019), estas condições também são expressas em termos de desigualdades de Lyapunov-Metzler derivadas da escolha de uma função de Lyapunov do tipo mínimo, e apresentam complexidade computacional similar a de Geromel and Colaneri (2006), onde estas desigualdades foram introduzidas pela primeira vez no âmbito de sistemas lineares com comutação a tempo discreto. A escolha deste tipo de função de Lyapunov gera condições de estabilidade prática menos conservadoras, no que se refere às condições de existência de um conjunto de atração, do que aquelas baseadas em funções quadráticas como, por exemplo Deaecto and Geromel (2017) e Deaecto and Egidio (2016). Dois exemplos ilustram a técnica de projeto apresentada, sendo o primeiro um exemplo acadêmico e o segundo um exemplo de aplicação prática em conversores CC-CC do tipo boost.
A notação utilizada é padrão. Para vetores ou matrizes reais, $\left({ }^{\prime}\right)$ refere-se ao transposto. Para matrizes simétricas, o símbolo $(\bullet)$ denota o respectivo bloco simétrico. Os símbolos $\mathbb{R}$ e $\mathbb{N}$ representam o conjunto dos números reais e naturais, respectivamente. $\mathrm{O}$ conjunto $\mathbb{K}=\{1, \cdots, N\}$ é composto pelos primeiros $N$ números naturais positivos. Para uma matriz simétrica, $X>0(X \geq 0)$ indica que a matriz $X$ é (semi-)definida positiva. O conjunto $\Lambda$ composto por vetores $\lambda$ tais que $\sum_{i \in \mathbb{K}} \lambda_{i}=1$ e $\lambda_{i} \geq 0$ é chamado de simplex unitário. A combinação convexa de matrizes $\left\{X_{1}, \cdots, X_{N}\right\}$ é denotada por $X_{\lambda}=\sum_{i \in \mathbb{K}} \lambda_{i} X_{i}$.

\section{FORMULAÇÃO DO PROBLEMA}

Considere o seguinte sistema afim com comutação

$$
\begin{aligned}
x[k+1] & =A_{\sigma[k]} x[k]+b_{\sigma[k]}, \quad x[0]=x_{0} \\
z[k] & =E_{\sigma[k]}\left(x[k]-x_{e}\right)
\end{aligned}
$$

em que $x[k] \in \mathbb{R}^{n_{x}}$ é o estado e $\sigma[k] \in \mathbb{K}$ é a sequência de comutação responsável por selecionar um dos subsistemas $\mathcal{G}_{i}:=\left(A_{i}, b_{i}, E_{i}\right), i \in \mathbb{K}$, como ativo a cada instante de tempo $k \in \mathbb{N}$. No nosso contexto, esta sequência é, na verdade, uma função $\sigma: \mathbb{R}^{n_{x}} \rightarrow \mathbb{K}$ a ser projetada de modo a guiar o estado $x[k]$ até um conjunto de atração $\mathcal{X}$, contendo o ponto de equilíbrio $x_{e} \in \mathbb{R}^{n_{x}}$ de interesse, e que seja tão pequeno quanto possível. Como ficará claro posteriormente, este conjunto é não-convexo e difícil de tratar diretamente. Logo, para minimizá-lo, a ideia adotada em Egidio and Deaecto (2019) consiste na minimização do volume de um elipsoide auxiliar contendo o conjunto $\mathcal{X}$. Os demais resultados da literatura que tratam este problema, veja Deaecto and Geromel (2017) e Deaecto and Egidio (2016), requerem que o ponto de equilíbrio $x_{e}$ pertença a um conjunto predefinido, dado por

$$
X_{e}:=\left\{x_{e} \in \mathbb{R}^{n_{x}}: x_{e}=\left(I-A_{\lambda}\right)^{-1} b_{\lambda}, \lambda \in \mathcal{S}\right\}
$$

com $\mathcal{S} \subseteq \Lambda$ composto por todos os $\lambda \in \Lambda$ tais que $A_{\lambda}$ é Schur estável. Esta imposição não está presente nos nossos resultados e é um fator essencial para que $x_{e}$ seja parcialmente conhecido. Isto permite que o projetista escolha as componentes de $x_{e}$ que devem ser fornecidas, formando um conjunto de índices $\mathcal{I} \subseteq\left\{1, \cdots, n_{x}\right\}$, enquanto as demais são consideradas variáveis de projeto a serem determinadas via processo de otimização. Para este fim, definimos agora uma estrutura para o vetor $x_{e}$ que, sem perda de generalidade, pode ser escrito como

$$
x_{e}=x_{e}^{*}+\hat{x}_{e}
$$

em que $x_{e}^{*} \in \mathbb{R}^{n_{x}}$ contém as componentes $i \in \mathcal{I}$ fornecidas pelo projetista e as demais nulas e $\hat{x}_{e} \in \mathbb{R}^{n_{x}}$ deve ser determinado via processo de otimização. Note que, neste caso, a $i$-ésima componente de $\hat{x}_{e}$ é nula sempre que $i \in \mathcal{I}$.

Definindo a variável de estado auxiliar $\xi[k]=x[k]-x_{e}$, obtemos o sistema equivalente a (1) dado por

$$
\begin{aligned}
\xi[k+1] & =A_{\sigma[k]} \xi[k]+\ell_{\sigma[k]}, \quad \xi[0]=x_{0}-x_{e}=\xi_{0} \\
z[k] & =E_{\sigma[k]} \xi[k]
\end{aligned}
$$

sendo $\ell_{i}=\left(A_{i}-I\right) x_{e}+b_{i}, \forall i \in \mathbb{K}$. Para este sistema, o ponto de equilíbrio de interesse é a origem $\xi=0$.

Para o nosso projeto de controle, adotamos a seguinte função de Lyapunov do tipo mínimo

$$
v(\xi)=\min _{i \in \mathbb{K}} \xi^{\prime} P_{i} \xi
$$


e a função de comutação dependente do estado associada

$$
\sigma(\xi)=\arg \min _{i \in \mathbb{K}} \xi^{\prime} P_{i} \xi
$$

A escolha da função de Lyapunov (5) permite encontrar condições de existência do conjunto de atração $\mathcal{X}$ menos conservadoras que as funções de Lyapunov quadráticas, como discutido em Egidio and Deaecto (2019).

O nosso objetivo é o projeto da regra de comutação (6) assegurando a estabilidade prática do sistema por meio da existência de uma região $\mathcal{Z} \subset \mathbb{R}^{n_{z}}$ de maneira que $z[k] \notin \mathcal{Z}$ implica em $\Delta v(\xi[k])=v(\xi[k+1])-v(\xi[k])<0$. A região $\mathcal{Z}$ dotada desta propriedade é chamada região de convergência. A seguir apresentamos nossos resultados principais a respeito deste problema de controle.

\section{RESULTADOS PRINCIPAIS}

As condições de estabilidade prática para o problema em questão são fornecidas pelo próximo teorema, baseadas em desigualdades de Lyapunov-Metzler, veja Geromel and Colaneri (2006). A subclasse de matrizes de Metzler considerada $\mathcal{M}$ é dada pelas matrizes $\pi_{j i}=\Pi \in \mathcal{M}$ tais que

$$
\pi_{j i} \geq 0,(i, j) \in \mathbb{K} \times \mathbb{K}, \sum_{j \in \mathbb{K}} \pi_{j i}=1, \forall i \in \mathbb{K}
$$

Ademais, para todo $i \in \mathbb{K}$ definimos as matrizes $P_{\pi i}=$ $\sum_{j \in \mathbb{K}} \pi_{j i} P_{j}$ que serão utilizadas a seguir.

Teorema 1. Considere o sistema (4) com $x_{e}^{*} \in \mathbb{R}^{n_{x}}$ e $\mathcal{I} \subseteq\left\{1, \cdots, n_{x}\right\}$ dados. Se existirem matrizes simétricas $S_{i}, T_{i j}$, matrizes $J_{i}$, uma matriz de Metzler $\Pi \in \mathcal{M}$, um vetor $\hat{x}_{e} \in \mathbb{R}^{n_{x}}$ e escalares $r, \gamma_{i}$, solução do problema de otimização

$$
\begin{gathered}
\inf _{S_{i}, T_{i j}, J_{i}, \Pi, \hat{x}_{e}, r, \gamma_{i}} r, \quad \text { sujeito a } \\
{\left[\begin{array}{cccc}
S_{i} & \bullet & \bullet & \bullet \\
0 & \gamma_{i} r & \bullet & \bullet \\
A_{i} S_{i} & \ell_{i} & J_{i}+J_{i}^{\prime}-\sum_{j \in \mathbb{K}} \pi_{j i} T_{i j} & \bullet \\
\gamma_{i} E_{i} S_{i} & 0 & 0 & \gamma_{i} I
\end{array}\right]>0, i \in \mathbb{K}} \\
\\
\end{gathered}
$$

com $\ell_{i}=\left(A_{i}-I\right) x_{e}+b_{i}$ e $x_{e}=x_{e}^{*}+\hat{x}_{e}$, então a função de comutação (6) com $P_{i}=S_{i}^{-1}, i \in \mathbb{K}$, assegura que a bola

$$
\mathcal{Z}=\left\{z \in \mathbb{R}^{n_{z}} \quad: z^{\prime} z \leq r\right\}
$$

é uma região de convergência para o sistema (4).

Prova. Em um instante arbitrário $k$ considere que $\sigma(\xi[k])=i \in \mathbb{K}$. De maneira a facilitar a leitura, a dependência temporal será omitida. De (5), obtemos

$$
\begin{aligned}
\Delta v(\xi) & =\min _{\kappa \in \mathbb{K}}\left(A_{i} \xi+\ell_{i}\right)^{\prime} P_{\kappa}\left(A_{i} \xi+\ell_{i}\right)-\xi^{\prime} P_{i} \xi \\
& =\min _{\lambda \in \Lambda}\left(A_{i} \xi+\ell_{i}\right)^{\prime} P_{\lambda}\left(A_{i} \xi+\ell_{i}\right)-\xi^{\prime} P_{i} \xi \\
& \leq\left(A_{i} \xi+\ell_{i}\right)^{\prime} P_{\pi i}\left(A_{i} \xi+\ell_{i}\right)-\xi^{\prime} P_{i} \xi \\
& =\xi^{\prime}\left(A_{i}^{\prime} P_{\pi i} A_{i}-P_{i}\right) \xi+2 \xi^{\prime} c_{i}+\rho_{i}
\end{aligned}
$$

com $c_{i}=A_{i}^{\prime} P_{\pi i} \ell_{i}$ e $\rho_{i}=\ell_{i}^{\prime} P_{\pi i} \ell_{i}$. Definimos o conjunto não-convexo $\mathcal{X}$ como sendo

$$
\mathcal{X}=\bigcup_{i \in \mathbb{K}}\left(\mathcal{E}_{i} \cap \mathcal{C}_{i}\right)
$$

onde $\mathcal{C}_{i}=\bigcap_{j \in \mathbb{K} \backslash\{i\}}\left\{\xi \in \mathbb{R}^{n_{x}}: \xi^{\prime}\left(P_{i}-P_{j}\right) \xi \leq 0\right\}$ é a região no espaço de estado onde o subsistema $i \in \mathbb{K}$ é escolhido por (6) e

$$
\mathcal{E}_{i}=\left\{\xi \in \mathbb{R}^{n_{x}}:\left(\xi-\mu_{i}\right)^{\prime} Q_{i}\left(\xi-\mu_{i}\right) \leq c_{i}^{\prime} Q_{i}^{-1} c_{i}+\rho_{i}\right\}
$$

para $\mu_{i}=Q_{i}^{-1} c_{i}$ e $Q_{i}=P_{i}-A_{i}^{\prime} P_{\pi i} A_{i}$ é definido a partir da quadrática do lado direito de (12). Impondo que $Q_{i}>0$, obtemos de (12) a propriedade $\Delta v(\xi)<0, \forall \xi \notin \mathcal{X}$. Ademais, da definição (14) temos que $0 \in \mathcal{X}$.

Neste ponto, note que $\mathcal{L}_{i}=J_{i}+J_{i}^{\prime}-\sum_{j \in \mathbb{K}} \pi_{j i} T_{i j}<P_{\pi i}^{-1}$ sempre que (10) é valida. De fato, aplicando o Lema do Complemento de Schur em relação a $S_{j}=P_{j}^{-1}$ em (10), temos que $T_{i j}>J_{i}^{\prime} S_{j}^{-1} J_{i}$ e, portanto

$$
\begin{aligned}
\mathcal{L}_{i} & <J_{i}+J_{i}^{\prime}-\sum_{j \in \mathbb{K}} \pi_{j i} J_{i}^{\prime} P_{j} J_{i} \\
& \leq\left(\sum_{j \in \mathbb{K}} \pi_{j i} P_{j}\right)^{-1}
\end{aligned}
$$

em que a segunda desigualdade vem do fato de que, para quaisquer matrizes quadradas $G$ e $R>0$, a desigualdade $R^{-1} \geq G+G^{\prime}-G^{\prime} R G$ é verificada, veja Geromel et al. (2007). Na verdade, a utilização do limitante inferior $\mathcal{L}_{i}$ não implica em conservadorismo visto que as escolhas factíveis $J_{i}=P_{\pi i}^{-1}$ e $T_{i j}=J_{i}^{\prime} P_{j} J_{i}+\varepsilon I$, com $\varepsilon>0$ arbitrariamente pequeno, implica em $\mathcal{L}_{i}=P_{\pi i}^{-1}+\varepsilon I$. Dado que a desigualdade (15) é valida, de (9) e (10) podemos escrever

$$
\left[\begin{array}{cccc}
P_{i}^{-1} & \bullet & \bullet & \bullet \\
0 & \gamma_{i} r & \bullet & \bullet \\
A_{i} P_{i}^{-1} & \ell_{i} & P_{\pi i}^{-1} & \bullet \\
\gamma_{i} E_{i} P_{i}^{-1} & 0 & 0 & \gamma_{i} I
\end{array}\right]>0, i \in \mathbb{K}
$$

que após ser multiplicada de ambos os lados pela matriz $\operatorname{diag}\left(P_{i}, 1, I, I\right)$ e aplicando o Lema do Complemento de Schur em relação aos seus dois últimos blocos matriciais, nos leva a

$$
\left[\begin{array}{cc}
E_{i}^{\prime} E_{i} & \bullet \\
0 & -r
\end{array}\right]<\gamma_{i}^{-1}\left[\begin{array}{cc}
Q_{i} & \bullet \\
-c_{i}^{\prime} & -\rho_{i}
\end{array}\right], i \in \mathbb{K}
$$

Multiplicando a desigualdade (17) pela esquerda por $\left[\begin{array}{ll}\xi^{\prime} & 1\end{array}\right]$ e pela direita pelo seu transposto, temos por $S$-procedure (veja Boyd et al. (1994)) que

$$
\left\{\xi \in \mathbb{R}^{n_{x}}: \xi^{\prime} E_{i}^{\prime} E_{i} \xi<r\right\} \supseteq \mathcal{E}_{i}, \forall i \in \mathbb{K}
$$

Finalmente, esta última inclusão assegura que para um $\xi \in \mathbb{R}^{n_{x}}$ arbitrário tal que $\sigma(\xi)=i \in \mathbb{K}$, temos que se $z \notin \mathcal{Z}$, definido em (11), então consequentemente $\xi \notin \mathcal{E}_{i} \cap$ $\mathcal{C}_{i}$ e, portanto, $\Delta v(\xi)<0$ o que demonstra que a bola $(11)$ é uma região de convergência para o sistema, concluindo a prova do teorema.

Alguns comentários a respeito deste teorema são pertinentes neste momento. Primeiramente, note que conjunto de atração $\mathcal{X}$ garantido pelo Teorema 1 está contido dentro da região correspondente à bola $\mathcal{Z}$ em $\mathbb{R}^{n_{x}}$ que pode não ser limitada em $\mathbb{R}^{n_{x}}$. Isto naturalmente acontece pois o posto de $E_{i}^{\prime} E_{i}$ é menor ou igual a $n_{x}$. Mesmo assim, a estabilidade prática é garantida pela existência do conjunto limitado $\mathcal{X}$. Ademais, vale ressaltar que o cálculo de componentes do ponto de equilíbrio através do processo de otimização só foi possível devido ao fato da desigualdade (9) ser linear em $\hat{x}_{e}$ (que forma $\ell_{i}$ ) e de que nenhuma outra imposição é feita sobre o ponto de equilíbrio $x_{e}$. 
Por fim, note que as restrições apresentadas pelo Teorema 1 são não-convexas devido ao produto de variáveis matriciais e escalares do problema de otimização. A fim de introduzir condições mais adaptadas para serem resolvidas por métodos de otimização imediatamente disponíveis, o seguinte corolário é apresentado.

Corolário 2. As condições de projeto apresentadas no Teorema 1 são válidas quando as desigualdades

$$
\left[\begin{array}{cccr}
S_{i} & \bullet & \bullet & \bullet \\
0 & r & \bullet & \bullet \\
A_{i} S_{i} & \ell_{i} & J_{i}+J_{i}^{\prime}-\sum_{j \in \mathbb{K}} \pi_{j i} T_{i j} & \bullet \\
E_{i} S_{i} & 0 & 0 & I
\end{array}\right]>0, i \in \mathbb{K}
$$

e (10) são simultaneamente satisfeitas.

Prova. Assumindo que (19) é satisfeita, multiplicamos ambos os seus lados por $\operatorname{diag}(I, \gamma, I, \gamma I)$ e reescrevemos $\left(S_{i}, T_{i j}, J_{i}\right)$ como $\left(\gamma \tilde{S}_{i}, \gamma \tilde{T}_{i j}, \gamma \tilde{J}_{i}\right)$ para algum $\gamma \in \mathbb{R}$ positivo. Multiplicamos a matriz resultante por $\gamma^{-1}$ e obtemos (9) $\operatorname{com} \gamma_{i}=\gamma, \forall i \in \mathbb{K}$ e $\left(S_{i}, T_{i j}, J_{i}\right) \rightarrow\left(\tilde{S}_{i}, \tilde{T}_{i j}, \tilde{J}_{i}\right)$ provando, assim, o corolário.

Apesar das desigualdades apresentadas pelo Corolário 2 serem mais restritivas pela escolha de $\gamma_{i}=\gamma, \forall i \in \mathbb{K}$, estas são mais simples de resolver visto que, para uma matriz de Metzler $\Pi \in \mathcal{M}$ dada, as restrições são LMIs e o problema (8) se torna bem adaptado para resolvedores já disponíveis na literatura, veja Boyd et al. (1994).

Logo, uma matriz $\Pi \in \mathcal{M}$ apropriada pode ser encontrada através de busca por $N^{2}-N$ elementos de tal matriz, como discutido em Egidio and Deaecto (2019) e Geromel and Colaneri (2006). Esta última referência propõe igualmente uma restrição de estrutura para a matriz $\Pi$ de forma a reduzir a busca de $N^{2}-N$ elementos a apenas 1 .

Por fim, note que apesar do conjunto $\mathcal{Z}$ ser uma região de convergência, ele não possui a propriedade de invariância, ou seja, $z\left[k_{0}\right] \in \mathcal{Z} \Rightarrow z[k] \in \mathcal{Z} \forall k>k_{0}$. Isto ocorre pois, apesar do operador diferença da função de Lyapunov ser negativo para todo $z \notin \mathcal{Z}$, nada impede que $v(\xi[k])$ aumente quando $z \in \mathcal{Z}$. De forma a encontrar um conjunto invariante para as trajetórias do estado, o seguinte teorema, retirado de Egidio and Deaecto (2019), é apresentado.

Teorema 3. Considere matrizes $P_{i}, i \in \mathbb{K}$, uma matriz de Metzler $\Pi \in \mathcal{M}$ e um vetor com estrutura $\hat{x}_{e} \in$ $\mathbb{R}^{n_{x}}$, soluções do problema de otimização apresentado no Teorema 1. Os escalares positivos $\nu, \beta_{i}$ e $\theta_{i j}, i \neq j$ que formam a solução do problema de otimização

$$
\begin{gathered}
\inf _{\nu, \beta_{i}, \theta_{i j}} \nu, \quad \text { sujeito a } \\
{\left[\begin{array}{cccc}
\beta_{i} P_{i}+\sum_{j \in \mathbb{K} \backslash\{i\}} \theta_{i j}\left(P_{i}-P_{j}\right) & \bullet & \bullet & \bullet \\
0 & & & \\
\beta_{i} P_{\pi i} A_{i} & \beta_{i} P_{\pi i} \ell_{i} & \beta_{i} P_{\pi i} & \bullet \\
P_{i} & 0 & 0 & P_{i}
\end{array}\right]>0}
\end{gathered}
$$

para todo $i \in \mathbb{K}$, garantem que o conjunto de nível

$$
\mathcal{V}=\left\{\xi \in \mathbb{R}^{n_{x}}: v(\xi)<\nu\right\}
$$

é uma região de convergência invariante.

Prova. A prova está disponível em Egidio and Deaecto (2019) e foi, portanto, omitida.

\section{EXEMPLOS NUMÉRICOS}

Apresentamos agora dois exemplos que avaliam a eficácia da técnica de projeto proposta. O primeiro, é usado para comparar as condições de projeto do Corolário 2 para diferentes escolhas da saída controlada. Já o segundo, consiste em um exemplo de aplicação prática, onde o objetivo é controlar a tensão de saída de um conversor CC-CC do tipo boost.

\subsection{Exemplo 1}

Considere um sistema afim com comutação a tempo discreto (1) dado pelas matrizes

$$
\begin{gathered}
A_{1}=\left[\begin{array}{rrr}
1.8 & 1.9 & 0.7 \\
-0.1 & 0 & -0.3 \\
0 & 0 & 0.1
\end{array}\right], b_{1}=\left[\begin{array}{c}
0.1 \\
0.1 \\
0
\end{array}\right], \\
A_{2}=\left[\begin{array}{rrr}
0 & -1.2 & 1.3 \\
0.4 & 1.7 & -1.5 \\
-0.2 & -1.9 & 1.6
\end{array}\right], b_{2}=\left[\begin{array}{c}
0 \\
-0.2 \\
0
\end{array}\right]
\end{gathered}
$$

Note que ambos os subsistemas são instáveis e não existe $\lambda \in \Lambda$ tal que a combinação convexa $A_{\lambda}$ seja Schur estável. Por este motivo as técnicas existentes na literatura que utilizam a função de Lyapunov quadrática não permitem assegurar a estabilidade do sistema, visto que a existência de combinação convexa estável é uma condição necessária em todas elas.

Resolvemos o problema de otimização (8) considerando as condições do Corolário 2 para $x_{e}^{*}=0$ e duas escolhas diferentes da saída controlada e do conjunto $\mathcal{I}$ das componentes de interesse do estado, listadas a seguir

C1: $E_{1}=E_{2}=\left[\begin{array}{lll}1 & 0 & 0\end{array}\right]$ e $\mathcal{I}=\{1\}$

C2: $E_{1}=E_{2}=\left[\begin{array}{lll}0 & 0 & 1\end{array}\right]$ e $\mathcal{I}=\{3\}$

Definindo uma matriz de Metzler

$$
\Pi=\left[\begin{array}{cc}
p & 1-q \\
1-p & q
\end{array}\right]
$$

para cada um dos casos foi realizada uma busca por $(p, q) \in$ $[0,1]^{2}$ de maneira a minimizar o raio $r$ da bola $\mathcal{Z}$ definida em (11). Para ambos os casos, a solução ótima deste problema foi encontrada para $(p, q)=(0,0)$ fornecendo os raios $r_{\mathrm{C} 1}=0.4809$ e $r_{\mathrm{C} 2}=0.0282$, respectivamente. Através das soluções obtidas associadas a cada uma destas escolhas, a regra de comutação (6) foi implementada e as trajetórias de estado obtidas partindo de $x_{0}=\left[\begin{array}{lll}10 & 10 & 10\end{array}\right]^{\prime}$ podem ser observadas na Figura 1 . No detalhe pode-se observar que, em regime permanente, os valores de $x_{1}$ foram menor para o caso $\mathrm{C} 1$ e de $x_{3}$, para $\mathrm{C} 2$, como sugere a teoria. Apresentamos também os respectivos sinais de comutação produzidos por (6) na Figura 2.

Destas simulações, foi possível concluir que a função de comutação proposta foi eficiente em estabilizar as trajetórias deste sistema quando diferentes componentes do estado foram escolhidas para compor a saída controlada, sobre a qual a região de convergência $\mathcal{Z}$ é definida.

\subsection{Exemplo 2}

Este exemplo de aplicação prática utiliza a metodologia apresentada a fim de limitar a frequência de comutação 

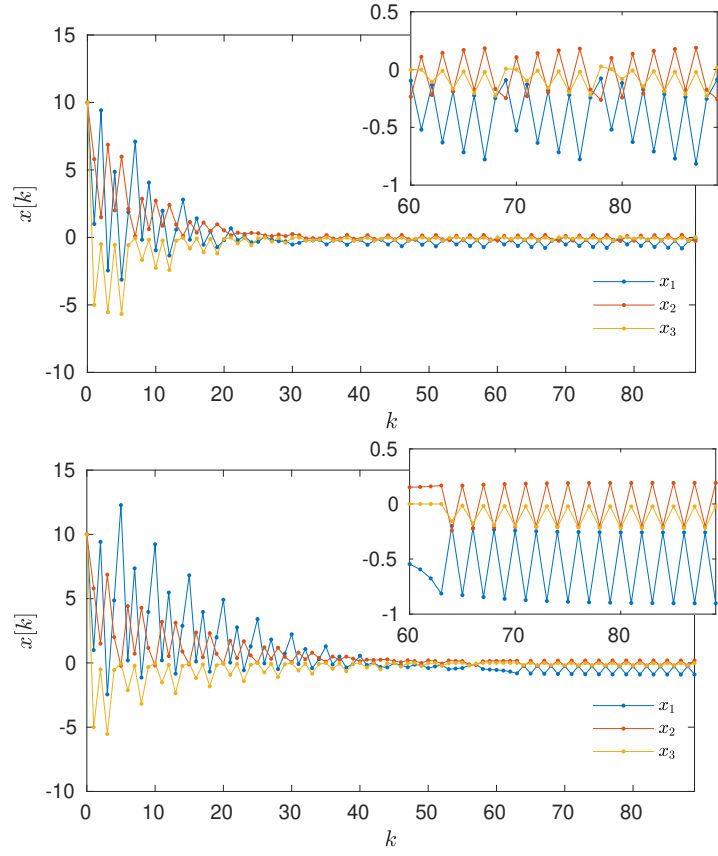

Figura 1. Trajetórias do estado para as regras de comutação obtidas para $\mathrm{C} 1$ e C2, respectivamente.
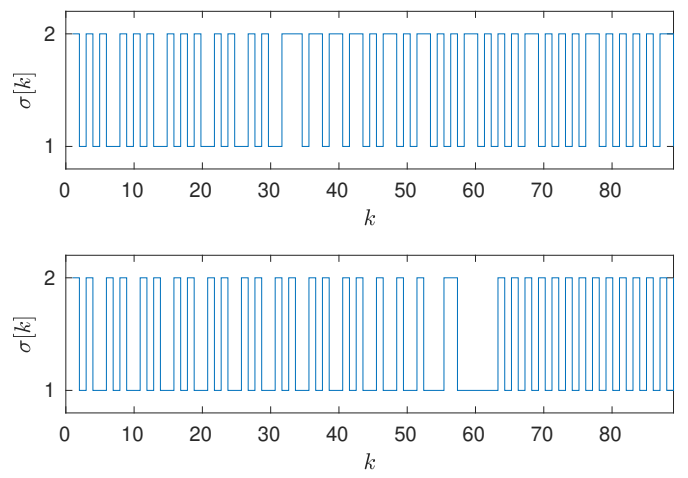

Figura 2. Sinais de comutação obtidos para C1 e C2, respectivamente.

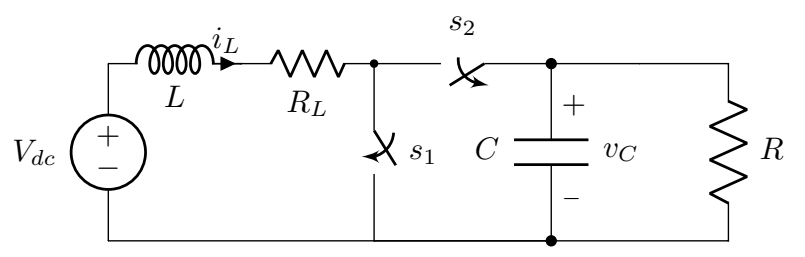

Figura 3. Esquemático do circuito boost.

das chaves de um conversor de potência CC-CC do tipo boost, esquematizado na Figura 3. Altas frequências de comutação são geralmente indesejáveis visto que estão associadas às perdas energéticas e, consequentemente, à eficiência do conversor.

Este sistema, recorrente em aplicações de eletrônica de potência, possui duas chaves $\left(s_{1}, s_{2}\right)$ eletricamente acionadas que são comandadas de maneira alternada, isto é, quando $s_{1}$ está fechada $s_{2}$ está aberta e vice-versa. O seu modelo dinâmico a tempo contínuo é dado por

$$
\dot{y}(t)=F_{\sigma(t)} y(t)+g
$$
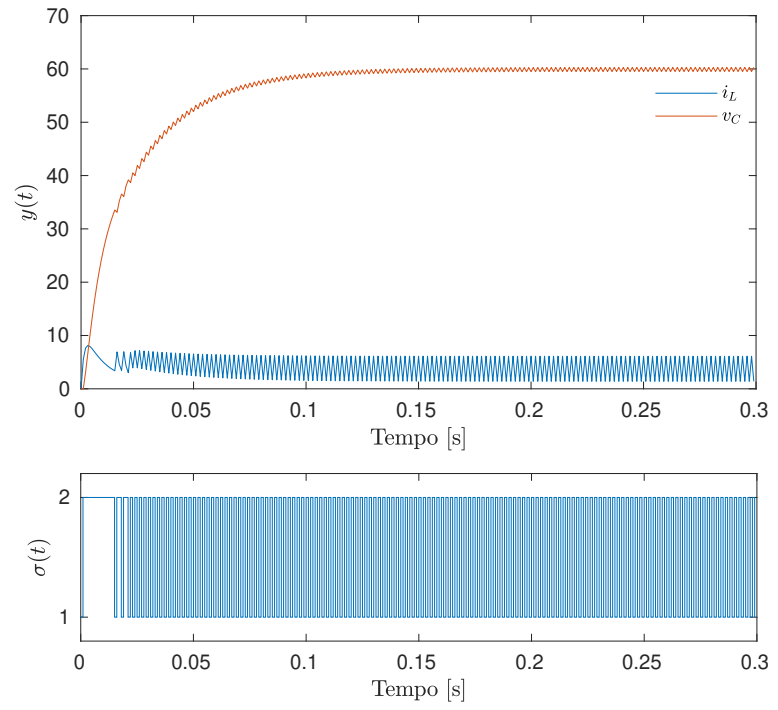

Figura 4. Trajetórias do estado do circuito boost e sinal de comutação associado.

sendo o estado $y=\left[\begin{array}{ll}i_{L} & v_{C}\end{array}\right]^{\prime}$ e as matrizes

$$
\begin{gathered}
F_{1}=\left[\begin{array}{cc}
-R_{L} / L & 0 \\
0 & -1 /(R C)
\end{array}\right], \\
F_{2}=\left[\begin{array}{cc}
-R_{L} / L & -1 / L \\
1 / C & -1 /(R C)
\end{array}\right], g=\left[\begin{array}{c}
V_{d c} / L \\
0
\end{array}\right]
\end{gathered}
$$

$\operatorname{com} V_{d c}=50 \mathrm{~V}, R_{L}=5.3 \Omega, L=6 \mathrm{mH}, C=2.2 \mathrm{mF}$ e $R=35 \Omega$. Adotando um período de amostragem $T_{a}=1$ ms e impondo que a regra de comutação deve se manter constante entre instantes de amostragem consecutivos $t_{k}$ e $t_{k+1}$, com $t_{k}=k T_{a}, k \in \mathbb{N}$, podemos reescrever este sistema no domínio do tempo discreto através do modelo (1) utilizando as matrizes $A_{i}=e^{F_{i} T_{a}}$ e $b_{i}=\int_{0}^{T_{a}} e^{F_{i} \tau} d \tau g$, $\forall i \in \mathbb{K}$. Isto garante que $y\left(t_{k}\right)=y\left(k T_{a}\right)=x[k], \forall k \in \mathbb{N}$.

Nosso objetivo é controlar a tensão de saída em $v_{C}^{*}=60$ $\mathrm{V}$ enquanto a corrente no indutor não é uma variável de interesse. Para este fim, definimos a parte fixa do ponto de equilíbrio desejado como $x_{e}^{*}=\left[\begin{array}{ll}0 & 60\end{array}\right]^{\prime}$ e o conjunto dos índices de interesse das componentes do estado como $\mathcal{I}=\{2\}$. Além disso, adotamos $E_{1}=E_{2}=\left[\begin{array}{ll}0 & 1\end{array}\right]$, definindo a região de atração $\mathcal{Z}=\left\{\left(v_{C}[k]-v_{C}^{*}\right)^{2} \leq r\right\}$.

Resolvendo o problema de otimização (8) com as condições do Corolário 2 para uma matriz de Metzler definida em (25) e realizando busca bidimensional em $(p, q) \in[0,1]^{2}$ encontramos para $(p, q)=(0,0)$ o valor $r=1.9395$, junto das matrizes

$$
P_{1}=\left[\begin{array}{cc}
0.3880 & 2.4040 \\
2.4040 & 18.0682
\end{array}\right], P_{2}=\left[\begin{array}{cc}
2.2010 & 5.9585 \\
5.9585 & 17.4515
\end{array}\right]
$$

e o vetor $\hat{x}_{e}=[5.64580]^{\prime}$ que permitiram a implementação da regra de comutação definida em (6). Simulando a resposta do sistema partindo de $y(0)=0$, obtivemos as trajetórias de estado e sinal de comutação apresentado na Figura 4. Além disso, a Figura 5 apresenta o plano de fase da parte final da trajetória $\xi[k]$ junto às superfícies de comutação $\mathcal{C}=\left\{\xi \in \mathbb{R}^{n_{x}}: \xi^{\prime}\left(P_{1}-P_{2}\right) \xi=0\right\}$ e às regiões de convergência $\mathcal{X}$ e $\mathcal{Z}_{\xi}=\left\{\xi \in \mathbb{R}^{n_{x}}: E_{\sigma} \xi \in \mathcal{Z}\right\}$. Notase que $\xi[k] \notin \mathcal{Z}_{\xi}$ implica na convergência do estado para $\xi=0$, como esperado. 


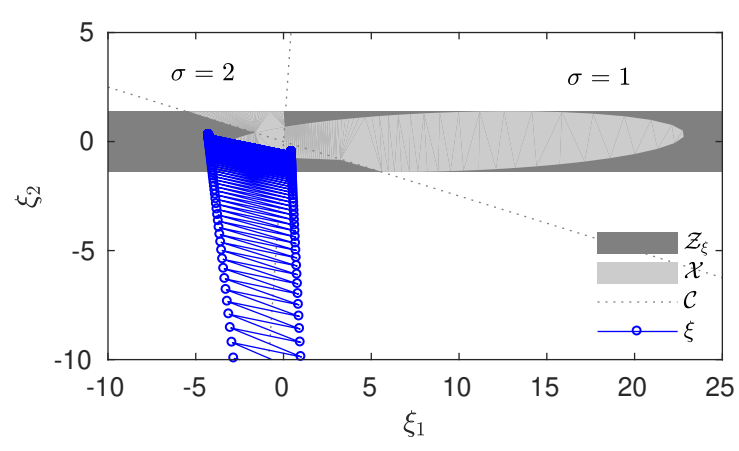

Figura 5. Regiões de interesse e $\xi[k]$ no espaço de estado.

Dessa forma, demonstramos o interesse prático da teoria apresentada, sendo possível controlar a tensão de saída do conversor CC-CC limitando a sua frequência de chaveamento.

\section{CONCLUSÕES}

Neste artigo, apresentamos condições de projeto para uma regra de comutação globalmente estabilizante capaz de garantir a existência de uma região de convergência $\mathcal{Z}$ no espaço vetorial da saída controlada $z[k]$. Esta regra garante que o estado converge para um ponto de equilíbrio de interesse $x_{e}$, sempre que $z[k]^{\prime} z[k]>r$, sendo $r$ uma variável de otimização. Ademais, diferentemente de outros resultados da literatura, nada é exigido em relação ao ponto de equilíbrio $x_{e}$ que também pode ser parcialmente fornecido. Um exemplo acadêmico, em que o sistema estabilizado não apresenta nenhuma combinação convexa estável entre seus subsistemas, foi apresentado. Por fim, a nossa metodologia foi aplicada em um conversor de potência CC-CC do tipo boost, demonstrando seu interesse prático.

Como trabalhos futuros, podemos listar a generalização destes resultados para tratar do caso de realimentação dinâmica de saída, incertezas politópicas e rastreamento de referências variantes no tempo.

\section{REFERENNCIAS}

Bolzern, P. and Spinelli, W. (2004). Quadratic stabilization of a switched affine system about a nonequilibrium point. In Proc. of the American Control Conference, volume 5, 3890-3895.

Boyd, S., Ghaoui, L., Feron, E., and Balakrishnan, V. (1994). Linear Matrix Inequalities in System and Control Theory. SIAM.

Camara, M., Gualous, H., Gustin, F., and Berthon, A. (2008). Design and new control of DC/DC converters to share energy between supercapacitors and batteries in hybrid vehicles. IEEE Transactions on Vehicular Technology, 57(5), 2721-2735.

Cardim, R., Teixeira, M.C.M., Assuncao, E., and Covacic, M.R. (2009). Variable-structure control design of switched systems with an application to a DC-DC power converter. IEEE Transactions on Industrial Electronics, 56(9), 3505-3513.

Deaecto, G.S. and Egidio, L.N. (2016). Practical stability of discrete-time switched affine systems. In Proc. of the European Control Conference, 2048-2053.
Deaecto, G.S., Geromel, J.C., Garcia, F.S., and Pomilio, J.A. (2010). Switched affine systems control design with application to DC-DC converters. IET Control Theory \& Applications, 4(7), 1201-1210(9).

Deaecto, G.S. (2016). Dynamic output feedback $\mathcal{H}_{\infty}$ control of continuous-time switched affine systems. Automatica, 71, $44-49$.

Deaecto, G.S., Fioravanti, A.R., and Geromel, J.C. (2013). Suboptimal switching control consistency analysis for discrete-time switched linear systems. European Journal of Control, 19(3), $214-219$.

Deaecto, G.S. and Geromel, J.C. (2017). Stability analysis and control design of discrete-time switched affine systems. IEEE Transactions on Automatic Control, 62(8), 4058-4065.

Deaecto, G.S., Geromel, J.C., and Daafouz, J. (2011). Dynamic output feedback $\mathcal{H}_{\infty}$ control of switched linear systems. Automatica, 47(8), 1713-1720.

Deaecto, G.S. and Santos, G.C. (2015). State feedback $\mathcal{H}_{\infty}$ control design of continuous-time switched affine systems. IET Control Theory \& Applications, 9(10), 1511-1516(5).

Egidio, L.N., Daiha, H.R., Deaecto, G.S., and Geromel, J.C. (2017). DC motor speed control via buck-boost converter through a state dependent limited frequency switching rule. In Proc. of the Conference on Decision and Control, 2072-2077.

Egidio, L.N. and Deaecto, G.S. (2019). Novel practical stability conditions for discrete-time switched affine systems. IEEE Transactions on Automatic Control. doi: 10.1109/TAC.2019.2904136.

Fiacchini, M., Girard, A., and Jungers, M. (2016). On the stabilizability of discrete-time switched linear systems: Novel conditions and comparisons. IEEE Transactions on Automatic Control, 61(5), 1181-1193.

Geromel, J.C. and Colaneri, P. (2006). Stability and stabilization of discrete time switched systems. International Journal of Control, 79(7), 719-728.

Geromel, J., Korogui, R., and Bernussou, J. (2007). $\mathcal{H}_{2}$ and $\mathcal{H}_{\infty}$ robust output feedback control for continuous time polytopic systems. IET Control Theory \& Applications, 1, 1541-1549(8).

Hespanha, J.P., Naghshtabrizi, P., and Xu, Y. (2007). A survey of recent results in networked control systems. Proceedings of the IEEE, 95(1), 138-162.

Hetel, L. and Fridman, E. (2013). Robust sampled-data control of switched affine systems. IEEE Transactions on Automatic Control, 58(11), 2922-2928.

Liberzon, D. (2003). Switching in Systems and Control. Birkhäuser Boston.

Scharlau, C.C., Oliveira, M.C., Trofino, A., and Dezuo, T.J.M. (2014). Switching rule design for affine switched systems using a max-type composition rule. Systems \& Control Letters, 68, $1-8$.

Shorten, R., Wirth, F., Mason, O., Wulff, K., and King, C. (2007). Stability criteria for switched and hybrid systems. SIAM Review, 49(4), 545-592.

Sun, Z. and Ge, S.S. (2005). Switched Linear Systems Control and Design. Springer-Verlag.

$\mathrm{Xu}, \mathrm{X}$. and Zhai, G. (2005). Practical stability and stabilization of hybrid and switched systems. IEEE Transactions on Automatic Control, 50(11), 1897-1903. 